\title{
Problemas en el diagnóstico del Síndrome de Reye
}

\author{
Dn.: D. Jiménez E." y A. Manterola A.**
}

\begin{abstract}
The clinical and laboratory antecedents of 13 cases with the Reye syndrome admitten in the Roberto del Rio Hospital during of the hepatic histopatholory to the diagnoeis.

In 8 patients the hepalical biopsy was consistent with the Reye syndrame, but there were no euch indications in the remaining five.

Comparing both groups no significative differences were found such as: previons desease to the huspitalization, como slage, time bet ween the begining of the desease and death and mortality. No significative differences were found in relatian to the preeence of vomita and impairment of respiratory tracts in the previous desesse; tieme of recuperation of the survivors; the rise of traneaminasas and glutamina, hipoprotrombinemia, hipoglicemia and hipoglucorraquia.

We conclued that although the hepatical histopahology separate Reye ayndrome patients in two froup there are not clinical or laboratory elements enough to characlerize each group. Since several sspects of the Reye syndrome are not elearly defined we think that the hepalical biopay is useful in theory and eventually a praclical contribution.
\end{abstract}

El Síndrome de Reye (S. de R.) es entendido como una encefalopatía aguda no inflamatoria asociada a una disfunción hepática, cuya traducción anatomopatológiea es una degeneración grase dífusa, trangitoria y severa del higado, e incluso de otras visceras. Esta definición excluye las bepatitis y las lesiones de onigen tóxico y/o sistémico con necrosis e inflamación centrolobulillar del hígado. ${ }^{\mathbf{1}} 2$

Esta asociación de encefalopatía aguda y hepatopatía se manifiesta con un cuadro clínico que habitualmente comprende:

a) Una enfermedad previa de vías respiratorias o gastrointestinal, seguida de vómitos que, por lo común, son muy notorios. Este cuadro previo puede ser una infección esporádica o pertenecer a una infección de tipo epidémico o estar condicionado a la ingestión de algún tóxico como alimentos contaminados con aflatoxina $B$ o hipoglicinas $\mathbf{A}$.

b) Compromiso neurológico traducido por alteración de conciencia de intensidad variable que al progresar lo hace por etapas susceptibles de ser graduadas, signos de hipertensión intracraneana y cambios electroencefalográficos correlacionables a la gravedad de la encefalopatía. EJ LCR es

\footnotetext{
*Becado de Pediatria en Hospilal Roberto del Ruo.

**Profeeor de Pedintra. Departamento de Pedielria, Fac. Medicina Santiago Norte, Universidad de Chile.
}

claro, con menos de 8 leucocitos $/ \mathrm{mm}^{3}$ y frecuente hipoglucorraquia, preferentemente en los niños menores.

c) Signos de disfunción metabólica intracelular por afección predominante del hepatocito- con liberación de enzimas citoplasmáticas especialmente transaminasas, creatinfosfoquinasas, etc.; y como reflejo de bloqueos intramitocondriales la acumulación de amonio, glutamina y otros metabolitos.

d) Trastornos generales del metabolismo tales como hipoglicemia, alteraciones acidobásicas e hidroelectrolíticas; y, por último,

e) Eventual presencia de compromiso de funciones hepáticas como hipoprotrombinemia y disminución de otros factores de coagulación, aunque rara vez ictericia.

De las publicaciones sobre el tema puede deducirse que el cuadro clínico y de laboratorio del S. de $R$. no tiene características patognomónicas, en efecto, sintomatología similar se ha observado en otros cuadros, incluyendo intoxicaciones por acetominofenos, salicílicos y disulfirán, encefalitis virales y secundario o alteraciones sistémicas severas en caso de shock, septicemias, anoxia severa, etc. No es de extrañar, por lo tanto, que en la literatura no exista un consenso acerca de los eriterios diagnósticos necesarios para definir el S. de R., entendido, éste, como una entidad clínica con un sustrato anatómico bien claro. 
Para algunos autores las consideraciones epidemiológicas, clínicas y de laboratorio, cuando están bien definidas, bastarían para el diagnóstico., 3,5 Para otros, en la actual etapa de conocimiento -cuando exísten muchas incógnitas por resolver respecto a la etiopatogenia- sería requisito del diagnóstico contar con una biopsia hepática oportunamente efectuada. ${ }^{6.7}$

En vista de esta discrepancia y para formarnos un criterio al respecto, revisamos todos los casos de S. de $R$. con estudio completo y que ingresaron al Hospital Roberto del Río en un período de 5 años, 1974 a 1978.

\section{MATERIAL Y METODO}

En esta revisión se analizan los antecedentes clíni$\cos y$ de laboratorio de los 13 pacientes con S. de R. a quienes, en el período de observancia, se efectuó estudio histopatológico del hígado por biopsia o en necropsia. En ellos estudiamos su edad, enfermedad previa al ingreso, preaencia de vómitos, compromiso de conciencia al ingresar y etapa del coma alcanzada durante la enfermedad, lapso de tiempo transcurrido entre el inicio de la sintomatologia y la recuperación. etapa más profunda del coma y fallecimiento, presencia de transamisas elevadas, hipotrombinemia, glutamina elevada, hipoglucorraquia, hipoglicemia y letalidad.

De acuerdo a las alteraciones anatomopatológicas encontradas en estos 13 pacientes se distinguen 2 grupos: un grupo, que incluye 8 nin̄os (grupo $A$ ), que presento degeneración grasa difusa, a gota fina, del higado y que, por lo tanto, consideramos como
S. de R. confirmados; $y$ otro grupo, al que pertenecen 5 pacientes (grupo B), en quienes la histopatología hepática no confirma el S. de R. ya que presentan otras lesiones asociadas a la esteatosis, en un caso erudado polimorfonuclear y en otro la necropsia mostró signos de meningoencefalitis o no presentan alteraciones hepáticas como en los tres casos restantes.

\section{RESULTADOS}

Al correlacionar la clínica con la histopatología y comparar ambos grupos se encuentran algunas diferencias en los siguientes aspectos (Cuadro l):

Los 13 pacientes, según la edad, se distribuyen entre 1 mes y 5 años, sin embargo, en el grupo Ao S. de R. confirmado casi todos los niños son mayores de 1 año, excepto 1 que tiene 5 meses, en tanto que en el grupo B o S. de R. inconfirmado, 3 de los 5 memenores de 6 meses.

La duración de la enfermedad previa al ingreso es más larga en el grupo A, con un promedio de 4 días, que en el grupo de S. de R. inconfirmado, con promedio de 2 dias. Destaca claramente, además, que el sindrome diarreico se presentó menos frecuentemente en el grupo A (2 de los 8) que en el grupo B (3 de los 5).

El compromiso encefálico, medido de acuerdo a las etapas del coma, grados 1 a 5 según Lovejoy, ${ }^{8}$ alcanzó grado 5 en sólo 3 de los 8 casos de $S$. de R. confirmados y, por el contrario, casi todos los pacientes del grupo $B$ llegaron al grado 5 ( 4 de los 5 ).

La enfemedad evolucionó a diferente velocidad en ambos grupos. Mientras en el grupo A transcu-

\section{CUADRO 1}

Diferencias clínicas en 13 casos de Síndrome de Reye confirmados y no confirmados por histopatología hepática

\begin{tabular}{|c|c|c|}
\hline & $\begin{array}{l}\text { Grupo A } \\
\text { Confirmados } \\
8 \text { cagos }\end{array}$ & $\begin{array}{l}\text { Grupo B } \\
\text { No confirmados } \\
5 \text { casos }\end{array}$ \\
\hline Edad menor de 6 meses & 1 & 3 \\
\hline $\begin{array}{l}\text { Enfermedad previa: } \\
\text {-Duración pronedio } \\
\text {-Símdrome diarreico }\end{array}$ & $\begin{array}{l}4 \text { dias } \\
2 \text { casos }\end{array}$ & $\begin{array}{l}2 \text { días } \\
3 \text { casos }\end{array}$ \\
\hline Etapa del coma alcanzeda & 3 & 4 \\
\hline $\begin{array}{l}\text { Dias entre inicio de enfermedad y: } \\
\text {-Etapa más profunda del coma alcanzada } \\
\text {-Fallecimiento }\end{array}$ & $\begin{array}{l}\text { 4,5 dias } \\
5 \text { días }\end{array}$ & $\begin{array}{l}2,5 \text { días } \\
3,2 \text { dias }\end{array}$ \\
\hline Mortalidad & 4 & 4 \\
\hline
\end{tabular}


rren 4,5 días entre el inicio de la enfermedad y la etapa más profunda del coma, en el grupo $B$ es de 2,5 días. Hasta el fallecimiento pasaron 5 días en el grupo confirmado y 3,2 días en el grupo $B$.

El pronóstico fue mejor para los niños del grupo $\mathrm{A}$ puesto que sobrevivieron 4 de $\operatorname{los} 8$; en cambio, fallecen 4 de los 5 niños con $S$. de $R$. no confirmado por $h$ histopatología. Esto es sólo parcialmente atribuible al hecho que a este grupo pertenezcar los niños más pequeños, ya que Huttenlocher y col. no encontraron mortalidad muy elevada en $\log S$. de $R$. en niños menores de 6 meses. ${ }^{9}$

No se encontraron diferencias entre ambos grupos en los siguientes aspectos clínicos y de laboratorio.

Tanto el compromiso de las vias respiratorias como los vómitos, en la enfermedad previa al ingreso, se presentaron con similar frecuencia ( 5 de los 8 del grupo A y 2 de los 5 del grupo B).

Al ingreso, la intensidad de la encefalopatia fue muy semejante en ambos grupos, hospitalizándose la mayoría en etapa 3 del coma ( 4 de los 8 del grupo confirmado y 2 de los 5 inconfirmados).

Los que sobrevivieron en ambos grupos se restablecen en 9 días promedio, contando el tiempo desde el inicio de la enfermedad.

De los resultados de laboratorio fueron constantes la elevación de las transaminasas, todas sobre 365 $\boldsymbol{\mu} / \mathbf{m l}$ e hipoprotrombinemia, cada vez que se midieron en ambos grupos. La glutamina aumentada sobre $15 \mathrm{mg} \%$ y la glucosa disminuida en plasma y LCR se observaron frecuentemente en ambos grúpos y en muy semejante proporción (en 4 de 5 del grupo A y 3 de 4 del grupo B).

\section{COMENTARIO}

Nuestra experiencia señala que aunque la histopatología hepática permite distinguir un grupo de pacientes en los que el $\mathrm{S}$. de R. se confirma, las diferencias encontradas con los pacientes con $S$. de $R$. sin confirmar no logran ser significativas, dado lo escaso de la casuistica; y, por el contrario, hay una cantidad de aspectos, tanto clínicos como de laboratorio, que no permitirían distinguir un grupo de otro (cuadro 2), imposibilitando tener una caracterización de cada uno que sea de utilidad en la clínica aplicada.

Dado que para el $\mathbf{S}$. de $\mathbf{R}$. no exigte aún un tratamiento específico, esta diferenciación no tendría mayor significación y el médico estaría confinado a tratar rigurosamente y en forma planificada las manifestaciones sintomáticas, hipertensión endocraneana, trastomos acidobásicos e hidroelectrolíticos e hipoglicenia, corrección de otras alteraciones de la homeostasis y la insuficiencia hepática. ${ }^{2}$ La remoción rápida de toxinas y la entrega de sustancias esenciales, incluso aquellas de la congulaciôn, justificaría el uso de la exsanguineotransfusión en todo paciente que tiende a agravarse, ${ }^{10}$ aunque al respecto no hay unanimidad de opiniones. ${ }^{11,12}$

En una etapa de conocimiento en que se requiere la mayor clarificación nosológica que sea posible, de un cuadro clínico que no está aún bien definido desde el punto de vista etiopatogénico, creemos que la biopsia hepática es útil, primordialmente en los aspectos teóricos y en el diagnóstico diferencial, concordando con aquellos autores que piensan que el

\section{CUADRO 2}

Semejanzas clínicas en 13 casos de Síndrome de Reye confirmados y no confurmados por histopatología bepática

\begin{tabular}{|c|c|c|}
\hline & $\begin{array}{c}\text { Grupo A } \\
\text { Confirmados } \\
8 \text { casos }\end{array}$ & $\begin{array}{c}\text { Grupo B } \\
\text { No confirmados } \\
5 \text { casos }\end{array}$ \\
\hline $\begin{array}{l}\text { Enfermedad previa: } \\
\text { - Vías respiratorias } \\
\text { —Vómitos }\end{array}$ & $\begin{array}{l}5 \\
5\end{array}$ & $\begin{array}{l}2 \\
2\end{array}$ \\
\hline Ingreso en etapa 3 del coma & 4 & 2 \\
\hline $\begin{array}{l}\text { Días entre inicio y recuperación de } \\
\text { los sobrevivientes }\end{array}$ & 9 dias & 9 días \\
\hline $\begin{array}{l}\text { Transaminasas elevadas e } \\
\text { hipoprotrombinemia }\end{array}$ & 6 de 6 casos & 3 de 3 casos \\
\hline $\begin{array}{l}\text { Glutamina elevada, Hipoglicemia e } \\
\text { Hipoglucorraquia }\end{array}$ & 4 de 5 casos & 3 de 4 casos \\
\hline
\end{tabular}


S. de R. necegita el máximo de precisión posible y separarlo de otros procesos aimilares y, luego, eventualmente alcanzar una connotación práctica, ya que en nuestra breve serie los pacientes con $S$. de $R$. confirmados tienden a mostrar un mejor pronóstico que los casos no confirmados.

Por otra parte se ha observado que errores congénitos de enzimas mitocondriales pueden dar origen a cuadros semejantes al $S$. de $R$. en niños pequeños, aun en recién nacidos, 12, 13, 14 y también hasta formás recurrentes. ${ }^{15}$ Esto ha conducido a sospechar que dentro de los casos con S. de R. existen individuos con defectos enzimáticos incompletos que se ponen en evidencia cuando este sujeto portador es expuesto a infecciones o tóxicos o ambos a la vez. $\mathrm{Si}$ este es el caso, al estudio histopatológico del hígado debe seguir el estudio bioquimico de las enzimas mitocondriales comprometidas. Esta investigación, naturalmente, es completamente necesaria en los niños con $S$. de R. recurrentes. ${ }^{6}$

\section{RESUMEN}

Se revisan los antecedentes clínicos y de laboratorio de 13 casos de Síndrome de Reye, ingresados en un período de 5 años en el Hospital Roberto del Rúo con el propósito de evaluar, el aporte que al diagnóstico significa, la histopatología hepática.

En 8 pacientes la biopsia hepática fue compatible con el diagnóstico de Síndrome de Reye y en los 5 restantes no.

Al comparar ambos grupos se encuentran algunas diferencias que no alcanzan a ser significativas, como enfermedad previa al ingreso, etapa del coma alcanzado, tiempo entre inicio de la enfermedad y fallecimiento y mortalidad. No se encontraron diferencias entre ambos grupos en la presencia de vómitos y compromiso de vías respiratorias en la enfermedad previa, tiempo de recuperación de los sobrevivientes, elevación de transaminasas y glutamina, hipo- protrombinemia, hipogticemia e hipoglucorraquia.

Concluimos que aunque la histopatología hepática separa dos grupos entre los pacientes con Síndrome de Reye estudiados, no hay suficientes elementos clínicos o de laboratorio que permitan caracterizar cada grupo. No estando aún bien definidos varios aspectos del Síndrome de Reye, creemos que la biopsia hepática es útil en lo teórico y eventualmente lograr una connotación práctica.

\section{REFERENCIAS}

${ }^{1}$ Reve, R.D.K, et al. Encephalopaty and falty degeneration of the vịcera. A disease entily in childhood. Lancet 2: 749, 1963 .

${ }^{2}$ Manterola, A. Manifestacinnes neuruläpicas en hepatopatias del niño. Pediatría (Stgo., Chile) 22: 291, 1979.

${ }^{3}$ Huttenlocher, P.R. et al. Reye's syndrome in infancy. Pedio trics 60: 709, 1977.

${ }^{4} \mathrm{Hall}$ er, J.S. More on the uncertainties concerning Reye's syndrome (letter). J. Pediat. 89: 1041, 1976.

${ }^{5}$ Samaha, FJ. et al. Reye's syndrome: clinical diagnuais and treatment with peritoneal dialisys. Pediatrics 53: 336, 1974

'Partin, J.C. Hepatic encephalopathy and Reye"s ayndrome. Po diat Ann. 6: 346, 1977.

'Schubert, W.K. Comentary: The diagnosia of Reye's syndrome. J. Pediat. 87: 867, 1975.

'Lovejoy, F.H. et al. Clinical staging in Reye's syndrome. Am. J. Dir. Child. 128: 36, 1974.

${ }^{9}$ Huttenlocher, P.R. Reye's syndrome. Relation of oul come to therapy. J. Pediat. 80: B45, 1972.

${ }^{10}$ Bobo, f.C. et al. Reye's syndrome: treatmeal by exchange tranefusion with especial reference to the 1974 epidemis in Cincinant, Obio. J. Pedial. 87: 881, 1975.

' 'Corey, L. et al. Reye's ayadrome: clinical practesion and evaluation of therapy. Pediatrics 60: 708, 1977.

12 Freeman, JM, et al. Congenital hiperammonemia, Arch. Neurol. 23: 430, ]970.

${ }^{13}$ Ebels, E.J. Neurupathological changed in a patient with carbamylphosphate synthetase deficiency. Arch. Dis. Child. 47: 47, 1972.

${ }^{14}$ Wich, $H$ et al. Variants of citrullinameia. Arch. Dis. Child. 48: $636,1973$.

${ }^{15}$ Van Caille, M. et al.Reye's syndrome. Relapsec and neuroloycal sequelae. Pediatrics 59; 244, 1977. 